\title{
Is right to try being tried? Using crowdfunding data to better understand usage of nontrial pre-approval access pathways
}

\author{
Jeremy Snyder*,1(i), Alison Bateman-House ${ }^{2}$ iD $\&$ Leigh Turner $^{3}$ (D) \\ ${ }^{1}$ Faculty of Health Sciences, Simon Fraser University, 8888 University Drive, Burnaby BC V5A 1S6, Canada \\ ${ }^{2}$ Division of Medical Ethics, Department of Population Health, Grossman School of Medicine, NYU Langone Health, New York, NY \\ 10016, USA \\ ${ }^{3}$ Center for Bioethics, University of Minnesota, Minneapolis, MN 55455, USA \\ *Author for correspondence: jcs12@sfu.ca
}

\begin{abstract}
Aim: The US FDA has two nontrial pre-approval access pathways: expanded access (EA) and right to try (RTT). Reports of successful RTT use are scarce, and the FDA has not yet published RTT usage data, yet proponents tout its utility. In the face of this discrepancy and a lack of transparency of usage statistics, our aim is to add to the limited understanding of RTT usage. Materials \& methods: We searched crowdfunding campaigns referencing 'expanded access', 'right to try' or 'compassionate use' since 2018. Results: We identified 26 EA campaigns, 29 RTT campaigns and two referencing both. Twenty one EA campaigns described being approved to receive access to the requested experimental medical product versus one RTT campaign. Conclusion: RTT is associated with poor understanding of nontrial pre-approval access. These campaigns suggest RTT is not offering a practical alternative to EA. Cost remains a significant barrier to these patients.
\end{abstract}

First draft submitted: 13 April 2020; Accepted for publication: 14 September 2020; Published online: 12 October 2020

Keywords: compassionate use $\bullet$ crowdfunding $\bullet$ expanded access $\bullet$ right to try

US patients with serious diseases or conditions for which approved therapies are at present unavailable, or for whom approved medical products are not working, sometimes elect to use nontrial pre-approval access regulatory pathways. This nontrial option, colloquially called 'compassionate use', is particularly important for individuals who are unable to access the experimental interventions they seek within US FDA reviewed and cleared clinical trials and are unable to wait until an approved product comes to the market. Difficulty accessing clinical trials can occur when individuals do not meet study inclusion and exclusion criteria, cannot travel to study sites, or when relevant trials have closed to accrual or not yet opened for enrollment.

In the USA there are two ways to use experimental medical products outside of clinical trials. The older nontrial pre-approval access pathway providing this option is expanded access (EA). This regulatory channel permits individuals, or groups of similar patients, nontrial access to investigational drugs, biologics and devices at any clinical study stage before regulatory approval. This pathway is limited to those who do not qualify for clinical trials, have no FDA approved therapeutic options, have been diagnosed with serious or life-threatening conditions, and for whom the proposed experimental intervention is considered to have a favorable benefit/risk ratio. EA requests involve cooperation of a physician, permission from the FDA and an institutional review board, and agreement by the sponsor developing the investigational product (normally a biopharmaceutical company) to make it available via the EA pathway [1].

Despite numerous changes to make EA more accessible and streamlined, critics charge that the pathway constrains personal autonomy and creates unnecessary bureaucratic hurdles for patients [2]. While the merits of these claims have been widely questioned, right to try (RTT) laws permitting nontrial pre-approval access without FDA oversight 
were passed by 41 of the country's 50 state legislatures and, in May 2018, RTT became federal law, thus creating a second pathway by which terminally ill individuals may access investigational drugs outside of clinical trials [3].

RTT is more restrictive than EA, both in what kind of investigational agents qualify and which patients are eligible. Qualifying unapproved medical products must be drugs that have completed a Phase I clinical trial and are under continued investigation in studies overseen by the FDA. Furthermore, access to RTT is restricted to terminally ill individuals (groups of similar patients are not permitted) unable to participate in FDA cleared clinical trials and who have no approved treatments options. In both RTT and EA, companies or other entities developing the investigational medical product may only charge patients the direct costs of the product and shipping; they are not permitted to profit from making investigational interventions available pre-approval [4]. Furthermore, these sponsors developing investigational interventions may deny requests for access; despite the language of a 'right' to try, patients have only the freedom to ask for access, regardless of pathway. Insurers are not required to pay for preapproval access-related costs, and most insurers do not provide health insurance coverage for such investigational products. The key differences that distinguish the RTT option from the older EA pathway is the lack of FDA oversight and, in most cases, removal of institutional review board involvement [5].

These pathways for nontrial pre-approval access to investigational medical products have received renewed attention during the rush to develop new interventions to prevent or help treat COVID-19. For example, the pharmaceutical company (Gilead Sciences, CA, USA) provided its investigational antiviral drug remdesivir to thousands of patients diagnosed with COVID-19 via the EA pathway [6]. We are aware of no COVID-19-related uses of RTT. Hydroxychloroquine, another potential COVID-19 treatment that received considerable public attention and political support in the USA, had previously been approved by the FDA for treatment of malaria, lupus and rheumatoid arthritis; as such, it may be prescribed off-label for any use. While any doctor can, in theory, prescribe it, numerous state pharmacy boards are temporarily limiting hydroxychloroquine prescriptions in order to prevent a run on supply that would jeopardize availability of the drug for those using it for its approved indications [7]. While the drug has been tested in clinical trials for this new indication, there has been a clamoring for access via EA among those unable or unwilling to participate in these trials. The FDA also issued an emergency use authorization for this drug that allowed pharmaceutical companies to donate hydroxychloroquine products to the US national strategic stockpile and local public health authorities [8]. These actions related to hydroxychloroquine do not utilize the RTT or EA pathways, but President Trump has spread confusion by rhetorically linking access to hydroxychloroquine and other investigational products to the RTT legislation he championed [9]. Adding to the muddle, while EA is, by law, only for unapproved medical products, in this tumultuous moment, companies and the FDA are sometimes allowing approved items to be distributed for free via EA [10].

RTT proponents including President Trump, who signed the legislation into federal law, claim that RTT meets widespread demand for increased and easier access to investigational products [11]. Despite such bold assertions, only seven confirmed reports of individuals accessing medical interventions via RTT have to date been documented [12]. While the FDA is tasked with collecting and publishing reports from sponsors providing access via RTT, it has not yet done so. In late July 2020, the FDA released a proposed rule concerning annual reporting to the FDA of the provision of investigational drugs via RTT. If approved without change, this rule would not result in the first such report on RTT use until well after the November 2020 presidential election. This delay would arise from the mandatory public commenting period on the proposed rule, time to finalize the rule, a period of 60 days after finalization before submissions must be made, and time for the FDA to produce and publish its report [13]. Until this report is produced, there will remain a striking discrepancy between the exceedingly limited data concerning how often the RTT pathway is used and pronouncements by President Trump and other members of his administration about the law's impact. This disjunction is concerning and creates the potential for uninformed decision-making by policymakers and the promotion of unrealistic expectations on the part of patients and caregivers hoping to utilize RTT to access experimental interventions.

In the absence of transparent reporting by companies or the FDA, systematic study of online crowdfunding campaigns provides one useful tool for examining the use of RTT and EA because any costs incurred to use these whether direct costs for investigational products or ancillary costs for services required to access these interventions - are rarely reimbursed by health insurers. Given often substantial out-of-pocket costs, some patients or their advocates choose to crowdfund to seek donations to cover expenses relating to pursuing their use of unapproved investigational products. While the study of crowdfunding campaigns is unlikely to identify all individuals pursing investigational medical products via the RTT or EA pathways, this approach has the potential to provide insight 
into the circumstances of at least some individuals pursuing these options and to increase transparency of an opaque situation.

\section{Materials \& methods}

The GoFundMe platform was selected for this analysis as it is the largest crowdfunding platform in the world, is based in the USA, and dominates the medical crowdfunding sector [14]. We searched for medical crowdfunding campaigns on GoFundMe that referenced 'right to try', 'expanded access' or 'compassionate use' and that discussed seeking an experimental medical intervention in the US through nontrial pathways. These searches were conducted using a database of crowdfunding campaigns that scraped campaign data and between April 2019 and April 2020. To capture recently added campaigns, additional searches were conducted using GoFundMe's native search function from February-April 2020. Data including the campaign title, campaigner name, campaign and update text, funding requested, funding received, number of Facebook shares and campaigner location were recorded for each campaign in a shared spreadsheet. This search process returned 79 campaigns referencing 'right to try' and 115 campaigns referencing 'expanded access' or 'compassionate use'.

We restricted our analysis of these campaigns to those initiated in 2018 and later in order to capture campaigns initiated before, during and after passage of the federal RTT law. The first author reviewed the remaining campaigns and recorded the recipient's underlying medical condition, the investigational product sought, and specific discussions of the RTT and/or EA pathways. Campaigns not referencing RTT or EA in the context of the US pathways for accessing investigational products were excluded. Cases where interpretation was uncertain were reviewed by the second and third authors and discussed until consensus was reached.

\section{Results}

This process identified 26 campaigns referencing EA for access to experimental interventions and 29 campaigns referencing RTT. Two campaigns referenced both pathways, for a total of 53 campaigns. Of the 26 campaigns referencing the FDA-administered EA pathway, 25 individuals (96.2\%) reported seeking access to an intervention through this pathway and $21(80.8 \%)$ described being approved to receive access. The most common interventions sought were ONC201 for diffuse intrinsic pontine glioma $(\mathrm{n}=7)$, an intervention using umbilical cord blood derived stem cells for autism spectrum disorder and cerebral palsy $(n=3)$ and VTS-270, lumbar intrathecal infusion of 2-hydroxypropyl- $\beta$-cyclodextrin, for Niemann-Pick type $C(n=3)$. Beneficiaries of these campaigns were often minors $(n=17,65.4 \%)$ and ill to the point of dying during the campaign $(n=6,23.1 \%)$.

Having received FDA approval to access the desired intervention via EA, these campaigns generally aimed, at least in part, to pay for expenses related to receiving the investigational product. Reported expenses included direct costs, which ranged from US $\$ 15,000$ for an infusion of stem cells at Duke University to US\$125,000$\$ 160,000$ for ONC201 and US\$700,000 for an antisense oligonucleotide (ASO)-based experimental intervention for amyotrophic lateral sclerosis (ALS). In other cases, as with VTS-270, the direct costs of the intervention were paid for by the sponsors of the products-in-development. However, even in these cases, to access these interventions campaigners faced significant expenses related to travel, accommodations, meals and other costs.

The 29 campaigns mentioning RTT told a very different story. Only 14 (48.3\%) of these campaigns identified a specific intervention being sought, and just one (3.4\%) clearly described the campaign recipient being granted access to an intervention via RTT. Campaigners most commonly sought the NurOwn stem cell intervention $(\mathrm{n}=6)$ for ALS; stem cell interventions $(\mathrm{n}=5)$ for kidney failure, muscular dystrophy, autism spectrum disorder, and ALS; and, in the sole account of someone successfully accessing an intervention via RTT, ERC-1671 for glioblastoma. As the vast majority of the reported uses of RTT have been for this product, this finding supports our approach to identifying fundraising campaigns for RTT. As with campaigns for access via EA, these campaigns sought funding for the direct costs of treatment, ranging from US $\$ 20,000$ for a stem cell infusion to US $\$ 170,000$ for ERC-1671 to US $\$ 300,000$ for NurOwn. Similarly, other campaigns discussed and sought help for travel and accommodation costs required to seek investigational products.

While stem cell products were sought in campaigns for both pathways, they were much more common in campaigns invoking the RTT pathway $(n=11,64.7 \%$ of named interventions) than the EA pathway $(n=4,18.2 \%$ of named interventions). Part, but not all of this discrepancy, is likely due to fact that the company developing the NurOwn stem cell investigational therapy for ALS floated the idea of using RTT to provide the product outside of trials and eventually did so for one patient, one of the namesakes of the federal RTT law [15]. 
What distinguished the campaigns that invoked RTT from those that mentioned EA is that the first generally used RTT rhetoric as vernacular for a general desire to access experimental interventions rather than as part of a well-defined process of gaining nontrial pre-approval access to an investigational product. For example, one campaigner described RTT as "a program that may offer medical trials and new cutting edge medications not yet available to the general public." Used in this expansive manner, references to RTT could be referring to EA or access to investigational interventions within clinical trials, rather than to RTT as a specific regulatory pathway for access. In some cases, these campaigners thanked President Trump for allowing access to experimental interventions via RTT without stating if they had actually pursued a specific intervention using this pathway, as with the campaigner who stated "Thanks to President Trump, and the 'right to try act', it is now possible for terminally ill patients to receive experimental treatment." Others, without indicating that they had pursued an experimental treatment via RTT, flagged what they viewed as shortcomings of RTT, including that it was limited to persons with terminal illness. Some of the unproven treatments sought, such as naturopathic treatments, were not investigational medical products that had completed Phase I trials and were still in FDA approved trials, and hence did not qualify for use under RTT.

\section{Discussion}

Our analysis of these crowdfunding campaigns supports three observations concerning the functioning of the RTT pathway as compared with the EA pathway, both in the broader context of individuals seeking access outside clinical trials to investigational medical products.

First, invocation of RTT often is associated with what appears to be less clear understanding of the functioning and requirements of this pathway or less specific plans for invoking it. More generally, references to RTT are associated with a desire for readier access to investigational products that may be borne from frustration from limited opportunities to access experimental treatments via other pathways. In most cases, campaigners saw RTT as opening an actual or potential path to accessing experimental interventions without indicating that they would qualify to use it or had identified a sponsor willing to provide a particular unapproved product. For example, the fact that patients seeking access to NurOwn were doing so via RTT was likely due to the fact that the company developing that investigational product had announced that it would be available to patients via RTT if a national law were enacted; however, after RTT became law, the company reneged on this promise for all but one patient [16]. Furthermore, most campaigners depicted RTT as offering newly-available access to investigational medical products, rather than demonstrating awareness that it was an alternative to the longstanding EA pathway created to provide patients with an option for nontrial pre-approval access. By comparison, campaigners mentioning EA had generally identified a specific investigational product to be made available by a specific sponsor and were actively using this pathway.

The greater presence of stem cell products in the RTT campaigns may indicate that this limited understanding of these pathways is situated within a broader public misunderstanding of the current stage of development of regenerative medicine. Moreover, individuals who were receptive to misleading messaging about the effect of RTT on the availability of drugs-in-development may also be similarly receptive to misleading messaging in the regenerative medicine field [17].

Second, many criticisms have been lodged against EA, including that it is too restrictive, time-consuming, and cumbersome. Justified or not, most critiques of EA build upon the assumption that it involves too much bureaucratic 'red tape'. RTT is then presented as a more streamlined alternative to EA, as it removes the necessity of FDA review of nontrial access requests. However, the campaigns we analyzed provided no support for the idea that RTT is overcoming these perceived barriers to access or offering a practical alternative to EA. Given that both pathways may impose substantial direct and indirect costs on patients and caregivers, we had expected to find considerable activity on crowdfunding platforms by those using both pathways. However, only the EA pathway shows such activity, with the campaigns referencing RTT limited to only one clearly successful request.

Finally, judging from the campaigns on GoFundMe, cost remains a significant barrier for some patients seeking nontrial access to investigational products, whichever pathway individuals decide to pursue. Practical impediments can include very large direct costs for the intervention, ranging from tens to hundreds of thousands of dollars. This is true for stem cell interventions, which often cost tens of thousands of dollars or more per treatment, possibly explaining their frequent appearance in these campaigns. Even when the sponsor waives these costs and provides an investigational product free of charge, daunting travel and accommodation expenses can make it difficult for recipients to access these interventions. This echoes the situation of patients participating in clinical trials: while the 
medicines and trial-related procedures are typically offered for free, a significant number of participants experience 'financial toxicity' from expenses necessitated by their participation in the trial, for example, travel and parking [18]. Thus, even if sponsors were barring from passing on their direct costs for investigational medical products used via EA or RTT to patients, or if insurers were obliged to cover such expenses, substantial financial barriers to accessing experimental interventions would persist.

One caveat that must be noted is that campaigns on GoFundMe may not accurately reflect costs associated with pursuing EA or RTT. While it is possible to document and analyze claims about expenses and desired donations, the crowdfunding website has no independent vetting of campaigners' financial needs. Furthermore, it is impossible to tell from analyzing crowdfunding websites how many patients are accessing investigational medical products by paying for them directly or by raising funds via online means.

\section{Conclusion}

While financial barriers to accessing experimental medical interventions exist for some, driving their use of crowdfunding activities, there is little evidence from the campaigns we analyzed that state or federal RTT laws are improving access to investigational products or providing individuals with the resources they need to overcome these significant economic barriers to access. Once the FDA's guidance on the annual reporting of RTT is finalized, we will, in time, have more complete insight into how many patients are accessing investigational interventions via RTT. Should that happen, we will know much more about how the RTT pathway is or is not working. The FDA anticipates limited utilization of the RTT pathway, predicting six sponsors to submit reports annually. This is in keeping with our analysis of crowdfunding campaigns on the GoFundMe platform, which demonstrates very little active use of RTT as a regulatory pathway for assessing investigational drugs outside of clinical trials. This finding is not supportive of proponents' claims that the passage of RTT legislation has resulted in substantial numbers of individuals using this pathway to access investigational products.

\section{Future perspective}

Publication of a proposed rule for annual summary reporting requirements for sponsors using RTT means that greater transparency on RTT usage is likely in the future. However, as these summary reports will be issued only on an annual basis and delayed until after the 2020 presidential election, additional, current perspectives on RTT usage like that provided here remain important. Furthermore, as this annual summary will focus on usage of RTT rather than patient perspectives on perceived barriers to accessing experimental treatments via RTT or EA, the use of patient-focused narratives, like those found in crowdfunding campaigns, is still needed to understand the effects of RTT legislation.

If the FDA's annual report confirms the findings here - that RTT is less used than EA and those seeking RTT use have less defined plans for doing so - then considerable public frustration with RTT is likely to be voiced. This is particularly the case given the public perception, and repeated invocation by proponents of this legislation, of the notion that RTT successfully broadened access to experimental treatments. As a result, new efforts to reduce regulatory restrictions on access to investigational products is likely in the future, even if these pose no realistic change of meliorating patient access to desired potential treatments.

\section{Author contributions}

J Snyder gathered and analyzed the data and wrote the manuscript. A Bateman-House analyzed the data and edited the manuscript. $\mathrm{L}$ Turner analyzed the data and edited the manuscript.

\section{Financial \& competing interests disclosure}

The authors have no relevant affiliations or financial involvement with any organization or entity with a financial interest in or financial conflict with the subject matter or materials discussed in the manuscript. This includes employment, consultancies, honoraria, stock ownership or options, expert testimony, grants or patents received or pending, or royalties.

No writing assistance was utilized in the production of this manuscript. 
Summary points

- Nontrial access to investigational products ahead of regulatory authorization is made possible through expanded access (EA) and right to try (RTT) pre-approval access pathways.

- RTT became law in 2018, but the US FDA has not yet published a summary report on its usage, and while public statements of such usage are scarce, there have been outsized claims of effectiveness made by the legislation's supporters.

- As insurance coverage of expenses related to investigational medical products used outside of clinical trials is limited and these products can be very expensive, we analyzed crowdfunding campaigns to develop a better understanding of usage of both pre-approval access pathways in those patients who cannot pay these costs out of pocket.

- 26 campaigns on GoFundMe referenced EA, 29 referenced RTT and two referenced both. However, 21 of the EA-related campaigns described being approved to receive access versus only one of the RTT-related campaigns.

- Direct costs for the desired experimental products ranged from US\$15,000 to $\$ 700,000$. Indirect costs such as travel expenses were also discussed.

- These findings suggest that those referencing RTT may have a comparatively poorer understanding of or less specific plans for utilizing nontrial pre-approval access than those referencing the EA pathway.

- RTT does not appear to be overcoming perceived barriers to accessing investigational products via the EA pathway.

- Cost, be it direct or indirect, is a significant barrier to accessing investigational products outside of clinical trials for some people.

- If these findings are confirmed by the FDA annual report once it is released, there may be public disenchantment with RTT and a renewed push for legislative efforts thought to offer greater access to investigational products.

\section{References}

1. Bateman-House A, Robertson CT. The federal right to try act of 2017 - a wrong turn for access to investigational drugs and the path forward. JAMA Intern. Med. 178(3), 321-322 (2018).

2. Brodrick M. Free to choose: a moral defense of the right-to-try movement. J. Med. Philos. 45(1), 61-85 (2020).

3. Lynch HF, Zettler PJ, Sarpatwari A. Promoting patient interests in implementing the federal right to try act. JAMA 320(9), 869-870 (2018).

4. McBride Folkers K, Bateman-House A, Roberston C. Paying for unapproved medical products. Wake Forest J. Law Policy(2020). https://scholarship.law.bu.edu/faculty_scholarship/1002

5. Chapman CR, Eckman J, Bateman-House AS. Oversight of right-to-try and expanded access requests for off-trial access to investigational drugs. Ethics Hum. Res. 42(1), 2-13 (2020).

6. Grein J, Ohmagari N, Shin D et al. Compassionate use of remdesivir for patients with severe COVID-19. N. Engl. J. Med. 382, 2327-2336 (2020).

7. Karlin-Smith S. States take unusual actions to control on-label drug supply amidst COVID-19 shortages. Pink Sheet (2020). https://pink.pharmaintelligence.informa.com/PS142071/States-Take-Unusual-Actions-To-Control-OnLabel-Drug-Supply-Amidst-C OVID19-Shortages

8. Grady D, Thomas K, Lyons PJ. 7 answers to questions about the malaria drug trump keeps pushing. NY Times (2020). www.nytimes.com/article/coronavirus-hydroxychloroquine-malaria.html

9. White House. Remarks by President Trump, Vice President Pence, and members of the coronavirus task force in press briefing. White House (2020). www.whitehouse.gov/briefings-statements/remarks-president-trump-vice-president-pence-members-coronavirus-task-fo rce-press-briefing-6/

10. Usdin S. Controversy over stretching expanded access to off-label uses. Biocentury (2020). www.biocentury.com/article/304445

11. Florko N. A year after trump touted right to try, patients still aren't getting treatment. STAT (2019). www.statnews.com/2019/01/29/right-to-try-patients-still-arent-getting-treatment/

12. Snyder J. Trump brags that he’s helping patients access medical 'miracles.' He isn't. Washington Post (2019). www.washingtonpost.com/outlook/trump-brags-that-hes-helping-patients-access-medical-miracles-he-isnt/2019/11/15/14690142-0 0df-11ea-8501-2a7123a38c58_story.html

13. Food and Drug Administration. Annual summary reporting requirements under the right to try act. Fed. Regist. (2020). www.federalregister.gov/documents/2020/07/24/2020-16016/annual-summary-reporting-requirements-under-the-right-to-try-act

14. Monroe R. When GoFundMe gets ugly. Atlantic (2019). www.theatlantic.com/magazine/archive/2019/11/gof undme-nation/598369/

15. Usdin S. Brainstorm considering right to try for ALS therapy. BioCentury (2018). www.biocentury.com/article/295956/brainstorm-considering-right-to-try-for-als-therapy 
16. Marques Lopes J. Brainstorm decides not to offer investigational ALS therapy nurown. ALS News Today (2018). https://alsnewstoday.com/2018/06/29/als-therapy-nurown-not-available-under-new-policy-brainstorm-decides/

17. Caulfield T, Sipp D, Murry CE, Daley GQ, Kimmelman J. Confronting stem cell hype. Science 352(6287), 776-777 (2016).

18. Chino F, Zafar SY. Financial toxicity and equitable access to clinical trials. Am. Soc. Clin. Oncol. Educ. Book 39, 11-18 (2019). 